[11] E. V. MoCollum et M. Davis, Journal Biol. Chem., 1915, 23, 247.

[12] A. F. Morgan, F. B. King, R. E. Boyden et V. A. Petro. Journal Biol., Chem., 1931, 90, 771.

[13] T. B. Osborne et L. B. Mendel. Journal Biol. Chem., 1915, 20, 351.

[14] M. Plommet. Communication personnelle.

[15] G. A. Ramsdeli et C. F. Hufnagel. XiIIe Congrès internat. de Laiterie, 1953, t. 3, 1025.

[16] W. D. Salmon. Alabama Agric. Exp. St. Ann. Rep., 1932, nº 43.

[17] C. Sлӧ sтröм. XIII'e Congrès Intern. de Laiterie, 1953, t. 3, 1005.

[18] C. Voegriin et J. W. Thompson. Publ. Health Rep., 1936, 51, 1429

[19] G. Zweig et R. J. Bцock. Journal Dairy Sc., 1953, 36, 427.

\title{
L'INSPECTION SANITAIRE DU LAIT
}

par

\section{G. THIEULIN et D. BASILLE}

(Suite.)

\section{II. - TECHNIQUES BACTÉRIOLOGIQUES POUR L'INSPECTION DU LAIT PASTEURISE ET SIGNIFICATION DES RÉSULTATS}

Si les causes les plus graves, mais non pas les plus fréquentes, de la mauvaise qualité hygiénique du lait pasteurisé résultent soit d'un chauffage insuffisant, soit d'une recontamination par du lait cru, et si l'inspection préventive est capable d'éviter la mise en vente et la consommation d'un tel lait, les méthodes classiques de contrôle n'en gardent pas moins, dans tous les cas, toute leur valeur.

En effet, à la suite du traitement thermique du lait, diverses souillures peuvent intervenir, dues aux manipulations, au matériel, au personnel, et seules des techniques bactériologiques sont alors à même de les révéler et d'en établir l'importance.

Il s'agit done, grâce à ces techniques, d'apprécier le résultat final, la résultante d'un ensemble d'opérations composant la pasteurisation, au sens large du terme.

Nous verrons ultérieurement que les résultats analytiques ainsi obtenus permettent une interprétation rationnelle, motivant une action profonde, étendue, durable, en raison des conditions particulières qui régissent la commercialisation du lait de consommation en nature.

Les épreuves bactériologiques à retenir doivent pouvoir s'inclure dans la réalisation d'un contrôle courant, journalier; -les résultats fournis doivent être objectifs, cependant que leur signification permettra les interprétations nécessaires pour justifier et imposer la conduite de l'inspecteur.

La mise en évidence de certains éléments pathogènes, comme Mycobacterium tuberculosis, les Brucella ou la toxine staphylocoe- 
cique, est longue et délicate. Elle ne peut se concevoir que dans le cadre d'enquêtes scientifiques, étrangères à l'objectif ici considéré, immédiat et pratique.

D'autres germes pathogènes comme les Salmonella pourraient être décelés assez aisément, mais il est plus indiqué de réaliser des épreuves d'exécution plus facile et plus rapide, répondant indirectement à la recherche de ces germes, et permettant en même temps, une appréciation moins spécifique, mais plus générale, de la qualité hygiénique du lait. Grâce à ces tests, les fautes techniques de la pasteurisation (au sens large), sont dénoncées par des "mouchards" dont le témoignage est systématiquement recherché. Parmi les épreuves qui ont été étudiées, proposées ou adoptées, mentionnons, en allant de la plus particulière à la plus générale, la recherche : $1^{\circ} \mathrm{d}^{\prime}$ Escherichia coli; $2^{\circ}$ des germes indologènes; $3^{\circ}$ des bactéries coliformes ; $4^{\circ}$ des microbes sulfhydrogènes ; $5^{\circ}$ le dénombrement des germes de toute nautre.

En France, le décret du 26 avril 1939 sur le contrôle des ateliers de pasteurisation, avait rendu officielles, d'une part, la numération des germes de toute nature, et, d'autre part, la recherche des coliformes.

Le décret du 21 mai 1955 (1), relatif aux laits destinés à la consommation humaine, abrogeant le texte précédent, a maintenu la numération des germes de toute nature, mais a substitué la recherche des germes indologènes à celle des coliformes.

La portée du témoignage recueilli grâce à l'analyse bactériologique variera toujours suivant la qualité du témoin, et, aussi, selon la méthode choisie pour l'interroger: selon qu'ils seront apportés par les indologènes ou par les coliformes, les renseignements obtenus sur la recontamination du lait pasteurisé ne concorderont pas rigoureusement; le résultat d'un dénombrement de flore totale dans un lait donné dépendra du milieu employé, de la température et de la durée d'incubation des cultures.

Ayant considéré les travaux déjà effectués, nous avons poursuivi, sur ces deux points (choix des épreuves et choix du mode opératoire), une expérimentation dont nous avons cru pouvoir présenter ci-après les modalités et les conclusions.

\section{A. - Coliformes, indologènes, sulfhydrogènes, colibacilles}

Les trois premiers de ces groupes microbiens sont complexes et artificiels, étant déterminés arbitrairement par le procédé d'analyse qui les identifie. Les critères pratiques adoptés sont des particularités métaboliques qui n'appartiennent en propre à aucune

(1) Journal officiel, $\mathrm{n}^{\circ} 136$, du 9 juin 1955. (Le Lait, 1955.) 
espèce microbienne et qui sont inconstantes dans un certain nombre d'espèces les possédant en principe. Ajoutons que ces particularités ne s'excluent pas mutuellement.

Le groupe des coliformes se caractérise pratiquement par l'aptitude à fermenter le lactose avec production de gaz.

Le groupe des indologènes trouve son unité dans la propriété de dégrader les peptones en produisant de l'indol.

Le groupe des sulfhydrogènes est déterminé par la production d'hydrogène sulfuré.

Ajoutons que, selon une modalité comparable, les colibacilles peuvent être pratiquement révélés par leur propriété de cultiver, en milieu phéniqué (ou boriqué), à une température normalement dysgénégique, soit $42-43^{\circ}$.

Dans 395 échantillons de lait pasteurisé (certifié ou ordinaire), nous avons recherché simultanément la présence des indologènes et des sulfhydrogènes par le moyen d'une seule culture pour chaque échantillon, à raison de $0 \mathrm{ml}$. 1 de lait (2) dans 10 millilitres du milieu suivant, de notre composition :

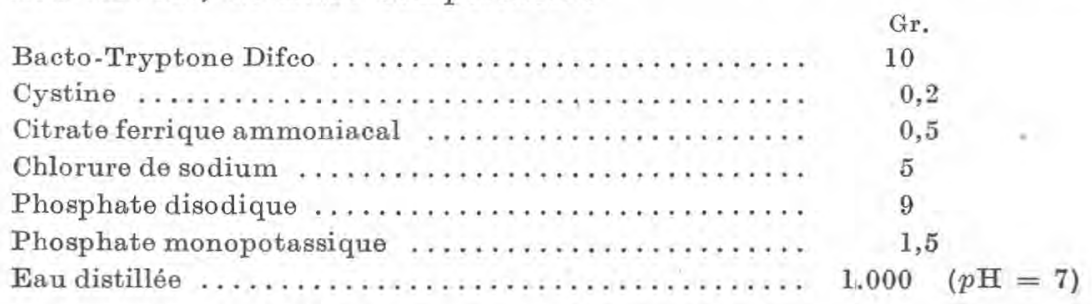

Après 48 heures d'incubation à $37^{\circ}$, la présence de sulfhydrogènes était dénoncée par un noircissement, soit diffus, soit localisé au culot de sédimentation qui se dépose dans les tubes; la présence des indologènes était révélée par addition du réactif d'Ehrlich-Kovacs.

Ce milieu ne correspond pas, pour la recherche des indologènes, à la formule officiellement adoptée par le décret du 21 mai 1955 (annexes) mais au cours d'une longue expérimentation antérieure, nous avions comparé notre eau peptonée, tamponnée, additionnée de cystine et de citrate ferrique, à l'eau peptonée simple " officielle ». Cette étude comparative, réalisée selon la méthode des 'ensemencements en double, toutes conditions égales d'ailleurs (et en particulier la peptone utilisée étant la même dans les deux milieux), nous avait permis de vérifier la concordance des résultats de la recherche des indologènes dans les deux milieux.

(2) Nous avons choisi ce volume de $0 \mathrm{ml}$. 1 de lait comme le plus indiqué (compte tenu du matériel étudié) pour obtenir un certain équílibre entre le nombre des résultats positifs et celui des résultats négatifs. Une étude comparative à sens unique eût perdu beaucoup de sa signification. 
A partir de chacun des mêmes 395 échantillons, fut réalisé un dénombrement des coliformes, par culture, en une plaque de gélose au désoxycholate de sodium, préparée à partir du bacto-désoxycholate-agar-déshydraté, Difco (1).

Le résultat du dénombrement des coliformes, réalisé sur 1 millilitre de lait, était rapporté, par calcul, à $0 \mathrm{ml}$. 1, pour permettre une comparaison avec les épreuves précédentes.

Les tableaux suivants mentionnent les résultats obtenus.

\section{TABLEAU I}

Sur 395 échantillons de lait pasteurisé examinés, les épreuves bactériologiques ont révélé la présence (dans $0 \mathrm{ml} .1$ de lait) :

a) de germes coliformes dans 182 échantillons soit $46 \%$ des laits ;

b) de germes indologènes dans 157 échantillons soit $40 \%$ des laits ;

c) de germes sulfhydrogènes dans 193 échantillons soit $49 \%$ des laits ;

d) de germes indologènes ou de germes sulfhydrogènes dans 225 échantillons soit $57 \%$ des laits.

\section{TABLEAU II}

Sur 213 échantillons ne contenant pas de coliformes dans $0 \mathrm{ml}$. I de lait (moins de 10 coliformes par millilitre), les épreuves bactériologiques ont révélé l'absence (dans $0 \mathrm{ml} .1$ de lait :

a) de germes indologènes dans 179 échantillons soit $84 \%$ des laits;

b) de germes sulfhydrogènes dans 167 échantillons soit $78 \%$ des laits;

c) de germes indologènes et de germes sulfhydrogènes dans 153 échantillons soit $72 \%$ des laits.

\section{TABLEAU III}

Sur 182 échantillons contenant au moins un coliforme par $0 \mathrm{ml}$. 1 de lait (10 coliformes au moins par millilitre), les épreuves bactériologiques ont révélé la présence (dans $0 \mathrm{ml} .1$ de lait) :

a) de germes indologènes dans 123 échantillons, soit $68 \%$ des laits;

b) de germes sulfhydrogènes dans 147 échantillons, sóit $81 \%$ des laits ;

c) de germes indologènes ou de germes sulfhydrogènes dans 165 échantillons, soit $91 \%$ des laits.

En résumé, la méthode des indologènes se montre, globalement, moins sensible que celle des coliformes pour le dépistage des contaminations du lait pasteurisé. Au contraire, la méthode des sulfhydrogènes, et, à plus forte raison, la méthode combinée indologènessulfhydrogènes, décèlent plus de contaminants que la méthode des coliformes.

La substitution de la méthode des indologènes à celle des coliformes (substitution consacrée par le décret du 21 mai 1955), aura pour effet de réduire la sévérité du contrôle. Cet allègement

(1) Difco laboratories Détroit, Michigan; U,S.A. (Distributeur en France : SEMP. 111, rue de l'Université, Paris). 
de la rigueur du contrôle avantagera, en premier lieu, les laits pasteurisés affectés de contaminations relativement discrètes, qui n'étaient dénoncées que "de justesse» par la méthode des coliformes ; les laits plus lourdement contaminés n’en bénéficieront qu’à un moindre degré.

Pour nous en convaincre, analysons la suite des 182 épreuves (tableau III ci-dessus) concernant les laits pasteurisés contenant au moins 10 coliformes par millilitre. Comme nous l'avons fait antérieurement, nous rapporterons le dénombrement des coliformes à $0 \mathrm{ml}$. I de lait, afin de rendre plus claire la confrontation. Nous avons vu que 123 seulement de ces 182 échantillons contenaient au moins 1 indologène par $0 \mathrm{ml}$. 1 et que 59 n'en contenaient aucun. Mais, si nous scindons cette suite d'épreuves en séries, ou paliers, délimités par le nombre de coliformes présents dans $0 \mathrm{ml}$. 1 de lait, nous constatons que la recherche des indologènes, dans le même volume du même lait, donne un résultat négatif pour :

\section{TABLEAU IV}

$62,5 \%$ (20 sur 32 ) des laits contenant de 1 à 1,9 coliformes par $0 \mathrm{ml} .1$; $51 \%$ (21 sur 41 ) des laits contenant de 2 à 4,9 coliformes par $0 \mathrm{ml} .1$; $27 \%$ (9 sur 33 ) des laits contenant de 5 à 9,9 coliformes par $0 \mathrm{ml}$. 1 ; $18 \%$ (6 sur 33 ) des laits contenant de 10 à 19,9 coliformes par $0 \mathrm{ml}$. 1 .

$7 \%$ ( 3 sur 43 ) des laits contenant plus de 20 colitormes par $0 \mathrm{ml} .1$.

Pour donner à notre étude comparative la rigueur désirable, il nous a semblé nécessaire de la compléter en confrontant, non plus des présences et des absences, mais des dénombrements. D'autre part, il convenait de vérifier, si, en se basant sur une technique différente, le dénombrement des coliformes et des indologènes se révélerait identique, et, d'apprécier enfin, à l'intérieur de ces groupes, la proportion de colibacilles.

Nous avons pour ce faire, utilisé les milieux suivants :

$1^{\circ}$ Le milieu vert brillant-lactose-bile que nous avons préparé à partir du Bacto-Brilliant-Green-Bile, Difco, déshydraté ;

$2^{\circ}$ Le bouillon lactosé au formiate et au ricinoléate de sodium (Bacto-Formate-Ricinoleate-Broth, Difco). Ce milieu est, comme le précédent, un milieu liquide pour la mise en évidence des coliformes : la présence de ces germes est révélée, dans les deux cas, par la fermentation gazeuse du lactose. Les gaz sont recueillis dans une petite cloche de Dunham, placée dans chaque tube;

$3^{\circ}$ La gélose lactosée au désoxycholate de sodium (Désoxycholate-Agar, Difco), milieu solide pour le dénombrement direct des coliformes ;

$4^{0}$ L'eau peptonée simple préparée selon la formule recommandée par le décret du 21 mai 1955. Ce texte n'ayant pas défini la 
peptone bactériologique exempte d'indol qui doit être utilisée pour l'élaboration du milieu, nous avons porté notre choix sur la bacto-tryptone, Difco ;

$5^{\circ}$ Le bouillon lactosé à l'acide borique (Boric-Acid-Broth, Difco), réplique de l'eau peptonée phéniquée couramment utilisée en France, en particulier pour la mise en évidence d'Escherichia coli dans les eaux. Dans les cultures en bouillon lactosé à l'acide borique, la détection des colibacilles ne repose pas sur la production d'indol, mais sur la fermentation gazeuse du lactose. Les cultures sont incubées, entre $42^{\circ} 5$ et $43^{\circ} 5$. Le volume du milieu doit être d'au moins 20 millilitres pour un inoculum de 1 millilitre.

Nous avons ainsi examiné 5 échantillons de lait pasteurisé (1), en réalisant, pour chacun, trois dilutions $(1 / 10,1 / 100,1 / 1.000)$ et 20 ensemencements dans chacun des quatre milieux liquides, soit 5 tubes ou fioles à 1 millilitre d'inoculum; 5 tubes à 0,$1 ; 5$ tubes à 0,01 et 5 à 0,001 . Seule la gélose au désoxycholate n'a exigé que

TABLEAU V

\begin{tabular}{|c|c|c|c|c|c|}
\hline \multirow[b]{2}{*}{$\begin{array}{l}\text { Numéro } \\
\text { de } \\
\text { l'échan- } \\
\text { tillon }\end{array}$} & \multicolumn{5}{|c|}{ Nombre de germes par millilitre de lait pasteurisé } \\
\hline & $\begin{array}{l}\text { Coliformes } \\
\text { en } \\
\text { vert-brillant }\end{array}$ & $\begin{array}{l}\text { Coliformes } \\
\text { en milieu } \\
\text { formiate- } \\
\text { ricinoleate }\end{array}$ & $\begin{array}{c}\text { Coliformes } \\
\text { en gélose } \\
\text { desoxycholate }\end{array}$ & $\begin{array}{c}\text { Indologènes } \\
\text { en eau } \\
\text { peptonée }\end{array}$ & $\begin{array}{c}\text { (1) } \\
\text { Colibacilles } \\
\text { en bouillon } \\
\text { lactosé } \\
\text { acide borique } \\
43^{\circ} \mathrm{C}\end{array}$ \\
\hline No 1 (2). & 28 & 14 & 16 & 0 & 0 \\
\hline $\mathrm{N}_{2}(3)$. & 24 & 92 & 60 & 0 & $11(7)$ \\
\hline No $3(4)$. & 92 & 160 & 59 & 7,2 & 0,7 \\
\hline No $4(5)$. & 1.600 & $\begin{array}{c}\text { p. de } 1.600 \\
(9)\end{array}$ & p. de 1.000 & 24 & 0,2 \\
\hline No $5(6)$ & 4,9 & 11 & 3 & 1,8 & 0 \\
\hline
\end{tabular}

(1) Test de présomption.

(2) Flore totale : 6.000 germes par millilitre de lait.

(3) Flore totale : 109.000 germes par millilitre de lait.

(4) Flore totale : 23.000 germes par millilitre de lait.

(5) Flore totale : 152.000 germes par millilitre de lait.

(6) Flore totale : 2.000 germes par millilitre de lait.

(7) Les 9 cultures positives $(5,3,1,0)$ ont été repiquées en eau peptonée. Aucune n'a donné naissance à un développement de germes indologènes.

(8) Dénombrement exact impossible en raison du trop grand nombre de colonies.

(9) Les 20 cultures étaient positives (production de gaz).

(1) Nous avons choisi à dessein des laits présumés contaminés (marques donnant habituellement des résultats défavorables). 
deux cultures en boîte de Pétri par échantillon : la somme des colonies dénombrées sur les deux plaques était, divisée par deux. Au total : 82 cultures pour chacun des 5 échantillons de lait.

Dans les milieux liquides, le dénombrement a été réalisé selon les règles classiques d'arrangement des séries de cultures positives et, le "nombre le plus probable" de bactéries était relevé dans la table de MaC Grady.

Les résultats sont consignés dans le tableau $\mathrm{V}$.

Dans l'ensemble, il apparaît nettement que les colibacilles ne composent qu'une faible partie des indologènes, eux-mêmes numériquement inférieurs aux coliformes. Cette comparaison peut permettre de mieux apprécier, quant à la qualité hygiénique réelle d'un lait examiné, les valeurs obtenues par l'une ou l'autre des techniques présentées.

Il serait, par exemple, faux de conclure que le nombre des coliformes correspond aux indologènes et, beaucoup plus encore, de déclarer que le nombre de l'un ou l'autre de ces groupes correspond à celui des colibacilles.

\section{B. - Dénombrement de la flore totale}

Par cette expression, il faut entendre le comptage des colonies obtenues dans des conditions expérimentales déterminées.

Nature du milieu de culture, température et durée d'incubation : tels sont les facteurs essentiels définissant l'épreuve.

En 1938, G. Guittonneau, G. Mocquot et A. Eyrard comparèrent un milieu, précédemment mis au point, qu'ils utilisaient couramment (à base de lait écrémé, digéré par la trypsine (1), à deux autres largement utilisés dans le monde : celui de BowERs et HuCKer (2) et celui de Demeter (3).

Les auteurs obtinrent avec ces trois milieux, en réalisant une incubation à $30^{\circ}$, des résultats pratiquement identiques.

Plus tard, G. GUittonneau et R. Chevalier simplifièrent la préparation du milieu à base de lait digéré, en remplaçant la papaïne par la trypsine, et obtinrent des résultats équivalents.

Le milieu défini par le décret du 21 mai 1955 (annexes) est une eau peptonée, gélosée, additonnée de glucose et de lait écrémé.

Nous avons comparé ce milieu, avant qu'il soit officiel, avec deux milieux commercialisés en France, sous forme déshydratée, l'un (Tryptone-Glucose-Extract-Agar, Difco) importé, l'autre fabriqué

(1) Taux final d'azote : $1,8 \%^{\circ}, p$ H $6,8-6,9$.

(2) Bacto tryptone $5 \mathrm{gr}$., bacto dextrose $1 \mathrm{gr}$, bacto agar $15 \mathrm{gr}$., lait écrémé $5 \mathrm{ml}$,, eau 1.000 .

(3) Extrait de viande Liebig $3 \mathrm{gr} .$, peptone $5 \mathrm{gr}$., lactose $10 \mathrm{gr}$., sel de cuisine $5 \mathrm{gr}$,, gélose 15 gr., eau distillée 1.000 . 
en France (milieu standard américain de l'Institut français de la fièvre aphteuse).

Pour que la comparaison avec le milieu officiel ne portât que les effets de l'adjonction de l'extrait de viande, toutes choses égales d'ailleurs, il ne fut ajouté à chacun des deux milieux déshydratés que 5 millilitres de lait écrémé par litre, au lieu de 10 généralement recommandés.

Dans ces conditions, 220 échantillons de lait pasteurisé furent ensemencés conjointement dans les trois milieux étudiés, à la dose de $0 \mathrm{ml}$. 001. Les cultures furent examinées après 48 heures d'incubation à $31^{\circ}$.

Les résultats d'ensemble conduisirent aux conclusions suivantes :

10 Les grosses différences dans le nombre des colonies développées respectivement sur chacun des trois milieux ensemencés avec le même matériel sont rares. Elles ne sont pas plus fréquentes ni plus accentuées que celles que l'on pourrait constater sur des séries de trois cultures sur le même milieu, à partir du même matériel ;

$2^{\circ}$ Si nous analysons la structure de cette suite de 220 épreuves en comparant entre elles les trois cultures issues de chacun des 220 échantillons, nous trouvons que :

a) le nombre de colonies développées sur le milieu déshydraté de fabrication française est, par rapport au nombre de colonies développées sur le milieu officiel,

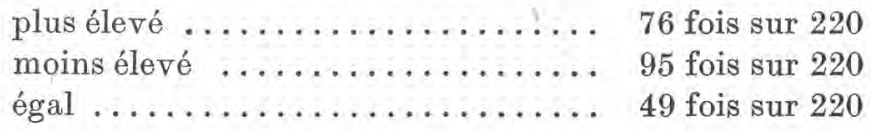

b) le nombre de colonies développées sur le milieu déshydraté importé des Etats-Unis est, par rapport au nombre de colonies développées sur le milieu officiel,

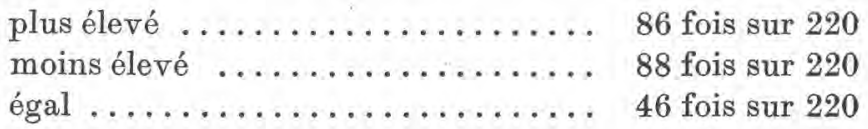

c) le nombre de colonies développées sur le milieu déshydraté de fabrication française est, par rapport au nombre de colonies développées sur le milieu déshydraté importé des Etats-Unis,

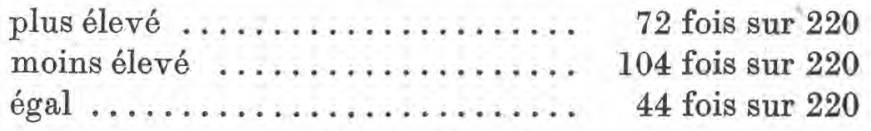

d) le nombre de colonies développées sur les trois milieux est, égal ............... 32 fois sur 220 ; 
$3^{\circ}$ Si nous tenons compte, non pas de la fréquence des divergences, comme nous venons de le faire, mais de leur importance en plus ou moins, par rapport au milieu de référence, nous nous apercevons qu'elles se compensent avec rigueur (voir tableau VI).

TABLEAU VI

\begin{tabular}{|c|c|c|c|}
\hline \multirow{2}{*}{$\begin{array}{l}\text { Résultat de } 3 \text { séries } \\
\text { de } 220 \text { cultures }\end{array}$} & \multirow{2}{*}{ Milieu officiel } & \multicolumn{2}{|c|}{$\begin{array}{l}\text { Gélose nutritive glucosée } \\
\text { deshydratée lactée }\end{array}$} \\
\hline & & $\begin{array}{l}\text { Fabriquée } \\
\text { en France }\end{array}$ & $\begin{array}{l}\text { Importée } \\
\text { des U.S.A. }\end{array}$ \\
\hline $\begin{array}{l}\text { Nombre total de colonies } \\
\text { développées dans les } 220 \text { cul- } \\
\text { tures } \ldots \ldots \ldots \ldots \ldots \ldots \ldots \ldots \ldots\end{array}$ & 5.732 & 4.448 & 4.672 \\
\hline $\begin{array}{l}\text { Nombre moyen de colo- } \\
\text { nies développées dans une } \\
\text { eulture } \ldots \ldots \ldots \ldots \ldots \ldots\end{array}$ & 21,5 & 20,4 & 21,2 \\
\hline
\end{tabular}

De nombreuses études, en particulier celles de Pederson et Yale, Robertson, Pederson et Breed, ont démontré que, dans l'ensemble, le dénombrement de la flore totale du lait donne un chiffre plus élevé lorsque la température d'incubation des cultures est de $30-31^{\circ}$, plutôt que $37^{\circ}$.

Le nombre des colonies développées dépend évidemment de l'optimum thermique des espèces bactériennes présentes dans le lait analysé.

Le degré de chauffage auquel est soumis normalement le lait pasteurisé français se situe très au-dessus du degré caractérisant la pasteurisation à l'étranger. Dans ces conditions, il nous a semblé intéressant d'apprécier, dans les conditions où nous nous trouvons, les variantes obtenues en incubant les cultures à $30-31^{\circ}$, au lieu de $37^{\circ}$.

Notre étude a porté sur 156 échantillons de lait pasteurisé, qui étaient ensemencés à la dose de $0 \mathrm{ml}$. 001, dans le milieu officiel, à raison de deux cultures par échantillon. L'une des boîtes de Pétri était placée à l'étuve à $37^{\circ}$ pendant 48 heures, l'autre à $31^{\circ}$ pendant le même temps.

L'ensemble des résultats s'exprime ainsi :

10 Le nombre de colonies développées à $31^{\circ}$ est, par rapport au nombre de colonies développées à $37^{\circ}$, 


plus élevé $\ldots \ldots \ldots \ldots \ldots \ldots \ldots \ldots \ldots \ldots \ldots \ldots \ldots \ldots \ldots \ldots \ldots$
moins élevé $\ldots \ldots \ldots \ldots \ldots \ldots \ldots \ldots$$\quad \begin{array}{r}115 \text { fois sur } 156 \\ \text { égal fois sur } 156\end{array}$

$2^{\circ}$ Lorsque le nombre de colonies développées à $37^{\circ}$ est supérieur au nombre de colonies développées à $31^{\circ}$, la différence est toujours petite;

$3^{\circ}$ Lorsque le nombre de colonies développées à $31^{\circ}$ est supérieur au nombre de colonies développées à $37^{\circ}$, la différence est parfois extrêmement grande ;

$4^{\circ} \mathrm{Si}$ l'on considère une longue suite d'épreuves, l'incubation à $31^{\circ}$ donne des dénombrements deux fois plus élevés que l'incubation à $37^{\circ}$, comme le montre le tableau VII ci-dessous.

\section{TABLEAU VII}

\begin{tabular}{c|c|c}
\hline \hline Résultat de deux séries de 156 cultures & \multicolumn{2}{|c}{ Température d'incubation } \\
\cline { 2 - 3 } & $31^{\circ} \mathrm{C}$ & $37^{\circ} \mathrm{C}$ \\
\hline $\begin{array}{r}\text { Nombre total de colonies développées } \\
\text { dans les } 156 \text { cultures } \ldots \ldots \ldots \ldots \ldots \ldots \ldots\end{array}$ & 5.099 & 2.476 \\
$\begin{array}{r}\text { Nombre moyen de colonies développées } \\
\text { dans une culture ................... }\end{array}$ & 32 & 16 \\
\hline \hline
\end{tabular}

D'un autre côté, il sera facilement admis que la durée d'incubation présente une grande importance et, pour les mêmes raisons et dans les mêmes conditions, nous avons jugé bon de réaliser une comparaison à ce sujet.

Nous avons ensemencé dans le milieu Tryptone-Glucose-ExtractAgar de Difco, 70 échantillons de lait pasteurisé, la dose d'inoculum étant de $0 \mathrm{ml}$. 001. Après 48 heures de séjour à l'étuve, à $31^{\circ} \mathrm{C}$, toutes les colonies visibles étaient pointées, puis, les boîtes de Pétri étaient replacées à l'étuve pour une durée supplémentaire de 24 heures. A l'expiration de ce délai, les colonies néoformées étaient dénombrées. Les résultats s'expriment dans le tableau VIII suivant.

En prolongeant de 24 heures la durée d'incubation, on a augmenté de $65,7 \%$ en moyenne le nombre des colonies.

Tout ceci nous montre que l'on ne peut songer raisonnablement à fixer des normes bactériologiques, en ce qui concerne en partieulier le dénombrement de la flore totale, sans apprécier en même temps, le milieu utilisé, la température d'incubation et la durée de cette incubation.

Une des «anomalies» du décret du 21 mai 1955 est d'avoir 
TABLEAU VIII

\begin{tabular}{|c|c|c|c|}
\hline \multirow{2}{*}{ Résultat de 70 eultures } & \multicolumn{2}{|c|}{ Durée de séjour à l'étuve } & \multirow{2}{*}{ Différence } \\
\hline & 48 heures & 72 heures & \\
\hline $\begin{array}{l}\text { Nombre total de colonies } \\
\text { développées dans les } 70 \text { cul- } \\
\text { tures } \ldots \ldots \ldots \ldots \ldots \ldots \ldots\end{array}$ & 2.090 & 3.465 & 1.375 \\
\hline $\begin{array}{l}\text { Nombre moyen de colo- } \\
\text { nies développées dans une } \\
\text { culture } \ldots \ldots \ldots \ldots \ldots \ldots \ldots\end{array}$ & 30 & 49 & 19 \\
\hline
\end{tabular}

précisément modifié la durée d'incubation, en vue de l'épreuve officielle, en portant de 48 heures à 72 heures cette durée, sans avoir en même temps modifié la norme, ou, tout au moins, considéré que cette norme, concernant le dénombrement avait été précédemment établie en tenant compte d'une durée d'incubation de 48 heures.

\section{CONCLUSION}

Nous disposons de techniques bactériologiques éprouvées, en vue de réaliser l'inspection du lait pasteurisé, et nous avons précisé - les modalités d'exécution, ainsi que les résultats pouvant être obtenus, de chacune d'elles.

Il importe d'accorder à chaque technique bactériologique sa signification réelle.

En présentant des résultats expérimentaux, nous avons voulu contribuer à établir que, pour éviter des interprétations erronées, spectaculaires, mais dangereuses, en même temps que susceptibles d'alerter gravement et à tort l'opinion publique, il convenait de connaître le sens exact des résultats obtenus.

Coliformes, indologènes, sulfhydrogènes, colibacilles, ne sont pas synonymes; le dénombrement de la flore totale doit être défini dans ses modalités d'exécution, et il est recommandé d'être bien informé pour ne pas risquer d'accuser à la légère un lait pasteurisé d'être pathogène.

(A suivre.)

\section{BIBLIOGRAPHIE}

G. Guittonneau, P. Sajou et R. De Peet. Recherche d'un milieu de culture approprié à la bactériologie fromagère. Le Lait, 1930, 10, 614 .

J. Pien, J. Bachimont et Firhol. Sur la recherche du Bacterium coli dans le lait. Le Lait, 1932, 12, 903, 1058 ; 1933, 13, 346, 548, 817.

G. Gutttonneau, G. Mocquot et A. Exrard. Recherches sur la pasteurisation des laits de consommation. I. Choix d'un milieu de culture pour 
la numération des germes microbiens. Le Lait, 1938, 18, 225. - II. Sur la colimétrie appliquée aux contrôles de la pasteurisation des laits pas. teurisés. Le Lait, 1939, 19, 113.

G. Guittonneau et R. Chevalier. Nouvelles techniques de préparation d'un milieu de culture à base de lait digéré ; le lait digéré par la papaïne. Le Lait, 1941, 21, 1.

Tanner. Microbiology of Foods. 2nd édition. Garrard Press, Champaign, Illinois, 1944, 320-334 / Bacterial Count of milk (bibliographie importante sur la numération des germes de toute nature).

G. Thieulin et R. Vuirladume. Eléments pratiques d'analyse et d'inspection

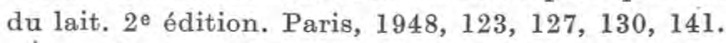

American Public Health Association. Standard methods for the examination of dairy products, 9th édition, New York, 1948.

S. -B. Thomas et J. M. Harcombe. Determination of the Coli-aerogenes content of milk by plating methods. Dairy Science Abstracts, 1954, 16, $n^{\circ} 8,608$. (Bibliographie importante sur le dénombrement des coliformes par cultures en milieux solides.)

G. Thieulin et D. Basille. Bul. Acad. Vet., 1955, n 7 .

\title{
DÉTECTION DES LAITS RECONSTITUÉS ET CHOIX D'UN RÉVÉLATEUR
}

\author{
par \\ V. TOUBOL \\ Ingénieur Chimiste I.C.N. \\ Directeur du laboratoire officiel de chimie de Casablanca \\ (Service de la Répression des Fraudes)
}

Les conditions économiques au Maroc ont suscité un intérêt exceptionnel dans la pratique d'une fraude simple à réaliser et pratiquement impossible à déceler.

Il s'agit de la substitution au lait frais d'un lait reconstitué à partir de lait en poudre écrémé, produit dont l'importation était tolérée pour la fabrication des aliments du bétail.

Si l'écrémage pouvait être décelé dans le cas d'une forte substitution, il n'en était pas de même pour une substitution de l'ordre de $20 \%$, car le lait des vaches marocaines a une teneur en matière grasse relativement élevée.

Voici quelques résultats authentiques sur plusieurs étables (déterminations faites au Laboratoire Officiel de Chimie, Service de la Répression des fraudes, en 1953) :

Matière grasse (en grammes par litre), de laits de mélange provenant d'étables différentes ou à différentes époques : $39,36,52$, $47,37,31,43,37,39,35,40,46,35,44,40,38,43,46,40,43,38$, 